\title{
National and Subnational Cancer Incidence for 22 Cancer Groups, 2000 to 2016: A Study Based on Cancer Registration Data of Iran
}

\author{
Javad Khanali $(\mathbb{D})$ and Ali-Asghar Kolahi \\ Social Determinants of Health Research Center, Shahid Beheshti University of Medical Sciences, Tehran, Iran \\ Correspondence should be addressed to Ali-Asghar Kolahi; a.kolahi@sbmu.ac.ir
}

Received 13 October 2020; Revised 10 May 2021; Accepted 29 June 2021; Published 13 July 2021

Academic Editor: Eleanor Kane

Copyright (C) 2021 Javad Khanali and Ali-Asghar Kolahi. This is an open access article distributed under the Creative Commons Attribution License, which permits unrestricted use, distribution, and reproduction in any medium, provided the original work is properly cited.

\begin{abstract}
Background. Cancer is an increasing public health concern, and detailed knowledge of the cancer incidence is required for developing effective cancer control plans. The objective of this study is to present the cancer incidence of 22 cancer groups in Iran and all 31 provinces of the country from 2000 to 2016, for both sexes across different age groups. Method. To study the national and provincial cancer incidence in Iran, we extracted data from the Cancer Project, which collects the Iranian cancer registry data and visualizes it in the VIZIT data visualization system. The methodology and statistical analysis that is used in this study follow the cancer project study protocol. Joinpoint analysis was performed to calculate the average annual percent change of the crude rates and age-standardized rates from 2000 to 2016. Results. Cancer incidence was 126,982 patients in 2016, and the crude rate (CR) of cancer in both sexes and all ages was 155 per 100,000 people. Cancer incidence approximately doubled between 2000 and 2016; however, the age-standardized rate (ASR) had a less drastic increase. The most incident cancers in 2016 were breast, skin, and colorectal cancers; however, the ranking of cancer groups by incidence was different in different age and sex groups and provinces. Some cancers exhibited a unique distribution pattern in the country with highincidence local areas. Discussion. The study showed that cancer incidence, crude rate, and age-standardized rate (ASR) in Iran had increased in 2000-2016 with vast heterogeneity by cancer type, province, and sex. Moreover, it was shown that the crude rate of cancer in Iran was much less than the global cancer crude rate. Providing such data helps to allocate resources and develop effective national cancer control plans appropriately.
\end{abstract}

\section{Introduction}

Cancer is an increasing public health concern [1]. Cancer incidence was 17.2 million patients worldwide in 2016, which shows a $28 \%$ rise since 2006. Cancer also imposed 213.2 million disability-adjusted life years (DALYs) on public health in the same year [2]. Moreover, it is estimated that global health will face 21.6 million patients a year by 2030 [3]. The increasing trend due to cancer will pose a threat to human development, necessitating developing action plans and political commitments to improve cancer control.

Quantitative assessment of the epidemiological measures on cancer would be mandatory for developing effective cancer control plans [4]. Providing such measures are more critical for developing countries like Iran that are facing many challenges in cancer management, such as rapid socioeco- nomic development and a growing and aging population [5-7]. Epidemiological measures on cancer are best provided by cancer registries; however, the pace of development of cancer registries is slow, and high-quality cancer data is not adequately available in many developing countries due to lack of human and financial resources [5]. A national pathology-based cancer registry program has been launched in Iran since 2000 by the ministry of health, covering all the 31 provinces of the country [8]. Although the overall completeness of the Iran cancer registry program is estimated between 60 and 75 percent, which is not ideal, it is a guide for Iranian policymakers to make more informed decisions about cancer control [7, 9-11].

Cancer incidence is an important epidemiological measure that could be used to estimate the overall effectiveness of health programs and required investment for cancer 
control. However, there is only a little published information regarding cancer incidence in Iran $[7,12]$. The objective of this study is to present the cancer incidence of specified 22 cancer groups in Iran in all 31 provinces of the country from 2000 to 2016 for both sexes and across all age groups. Herein, we reanalyzed the existing data of the cancer registration program of Iran and implemented data presentation methods used by the Global Burden of Disease (GBD) $[2,4,13]$.

\section{Material and Methods}

2.1. Data source. To study Iran's national and provincial cancer incidence, we extracted data from the Cancer Project, which collects the Iranian cancer registry data and visualizes it in the VIZIT data visualization system [14]. The national cancer registry data is collected from pathology centers, hospitals, and death registries [15]. The main data that is obtained from each cancer case include identification characteristics, age, gender, place of residence, codes of diagnosed cancer, and year of initial diagnosis [15]. Since the first available published report of the cancer registry in Iran dates back to 2000 [16], we limited the study period to the years 2000 to 2016.

The number of cancer incidence cases at the global level and in each sociodemographic index (SDI) quintile was extracted from the supplemental content of the 2016 Global Burden of Disease Study [2]. SDI is a composite measure of development status, which is strongly correlated with health outcomes. The index is a geometric mean of the income per capita, average educational attainment, and fertility rates of specified regions [17].

2.2. Definition. Classification of the cancer types was defined by the International Statistical Classification of Diseases and Related Health Problem 10th Revision (ICD-10). Twenty-two cancer groups were included in the study, and eTable 1 represents specified ICD-10 codes allocated to each group. The classification of cancer groups in VIZIT and the 2016 GBD study was matched with the defined cancer groups of our study.

2.3. Statistical analysis. The methodology and statistical analysis that is used in this study for calculating cancer incidence, crude rate (CR), and age-standardized rate (ASR) follow the cancer project study protocol $[18,19]$. This project was designed to estimate cancer incidence in Iran and provides data on cancer incidence, crude rate, and age-standardized rate for 70 subtypes of cancer in different age groups from 1990 to 2016 [19]. For calculating the age-standardized rate, the direct method of standardization was implemented, and the population of Iran in 2016 was used as the standard population. Joinpoint analysis was performed using Joinpoint Trend Analysis Software (Version 4.9.0.0) to calculate the average annual percent change of crude rate and agestandardized rate for each cancer group from 2000 to 2016.

\section{Results}

3.1. National Cancer Incidence. The incidence of cancer was 126,982 patients, and the crude rate (CR) for both sexes was 155 per 100,000 people in 2016 (Table 1 ). The most common cancer groups in both sexes combined were breast (14568 cases), skin (11712 cases), and colorectal cancers (11422 cases) (Table 1$)$. In women, breast cancer was by far the most common cancer (14217 cases) and accounted for $22 \%$ of all cancer cases. Colorectal (5717 cases) and skin cancers (5354 cases) were the second and third most common cancers in women, respectively. In men, the most common cancers were prostate (7963 cases), skin (6357 cases), and stomach cancers (5477 cases), which altogether accounted for $30 \%$ of all cancer cases.

Cancer incidence approximately doubled between 2000 and 2016, and an increase in the number of incident cases occurred in all cancer groups except esophageal cancer. The average annual percent change of the crude rate (CR) was significantly positive in both sexes for 14 out of 21 specified cancer groups. Stomach cancer was the only cancer with a different trend of CR in women and men, which was increasing for women but decreasing in men (Table 1). The annual percent change in both CR and ASR was higher in women than men (Tables 1 and 2 ). The age-standardized rate (ASR) also increased from 146.4 per 100,000 in 2000 to 155 per 100,000 in 2016. The ASR of cancer in women had an increasing trend in 26 provinces, while in men, only 16 provinces showed an increased ASR (Table 2). Markazi, Hormozgan, and Qazvin showed the highest annual percent change of ASR for women among all provinces. For men, Markazi, Bushehr, and Hormozgan showed the highest ASR annual percent change. Zanjan, Kerman, and Lorestan were the only provinces with a decreasing trend in cancer ASR for both women and men (Table 2, Figure 1).

Cancer incidence showed a heterogeneous distribution in Iran. For instance, cancer ASR varied from 219.7 in Khuzestan to 74.7 in Sistan and Balouchestan in 2016 (Figure 2, Table 2). The seven provinces that their cancer ASR was higher than the national average were Khuzestan, Yazd, Fars, Tehran, Khorasan Razavi, Isfahan, and Semnan (Table 2).

The ranking of cancer groups by incident cases was different between the global and national levels (Figure 3). For instance, the "trachea, bronchus, and lung" cancer group has the highest incidence worldwide; however, it was ranked seventh in Iran. As another example, cervical cancer was the eleventh most incident cancer worldwide; however, it was the least incident cancer group in Iran. Likewise, the incidence of cancer groups in Iran was also heterogeneous. For instance, esophageal cancer was ranked tenth in Iran, but it was the most incident cancer in the North and South Khorasan. Despite such differences, the seven most common cancers in Iran were similar to the seven most common cancers globally, including breast, skin, colorectal, stomach, prostate, hematopoietic, and lung cancers.

The rank of cancer groups by incidence was different between different SDI quintiles. For example, cervical cancer had the highest incidence in low-SDI countries; however, it is nineteenth cancer by incidence in high-SDI countries. The pattern of cancer groups' ranking by the number of incident 


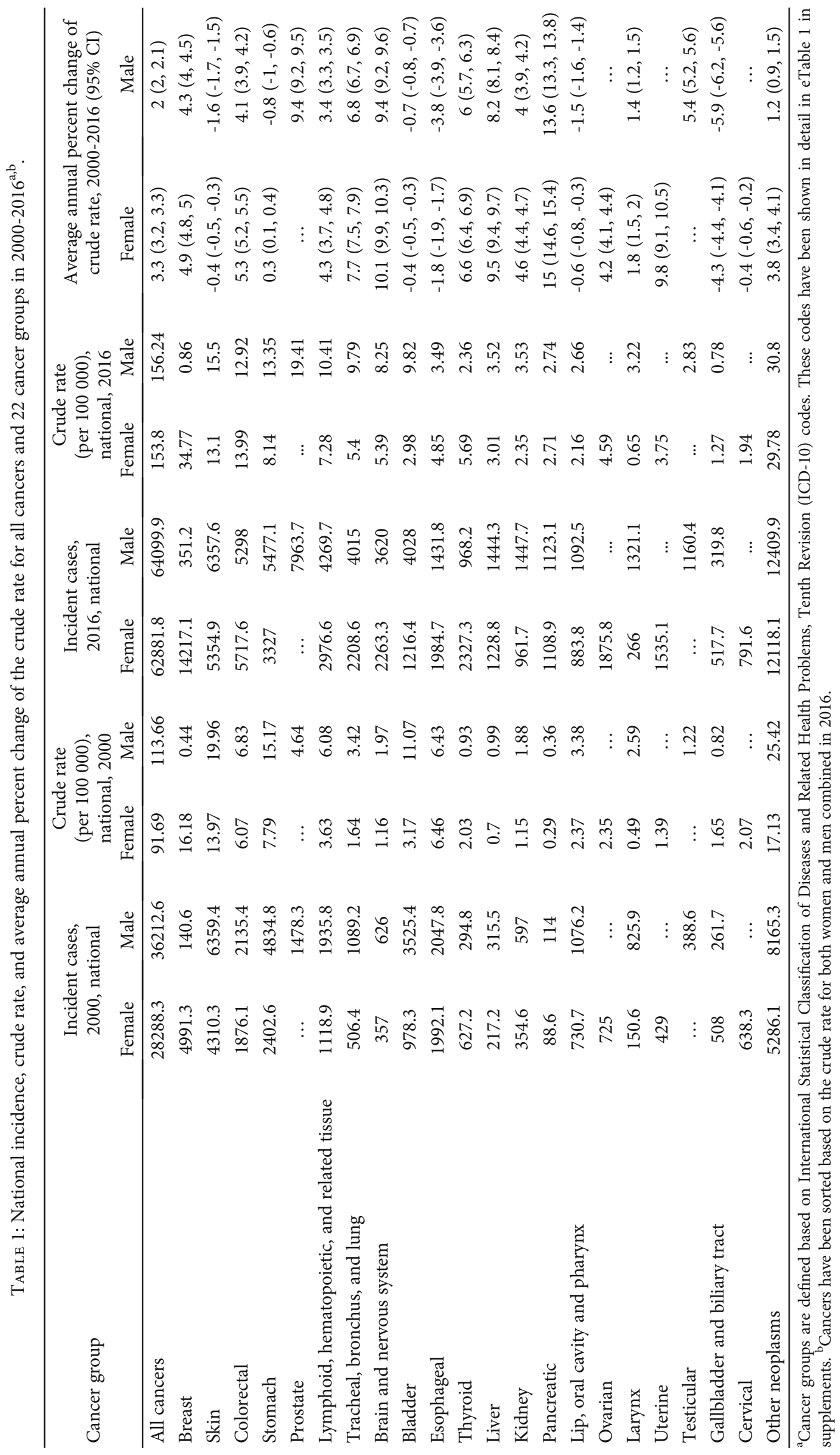




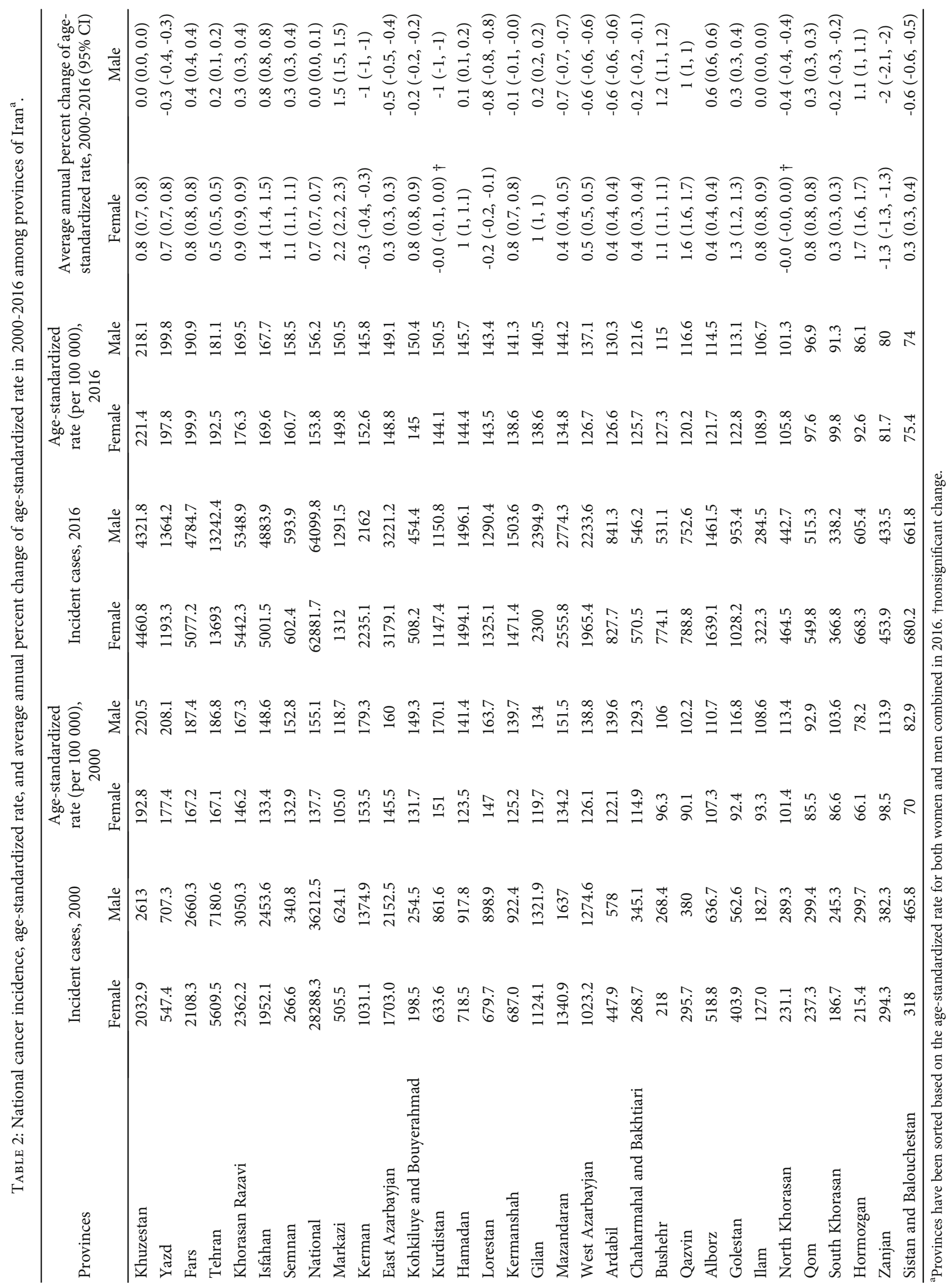




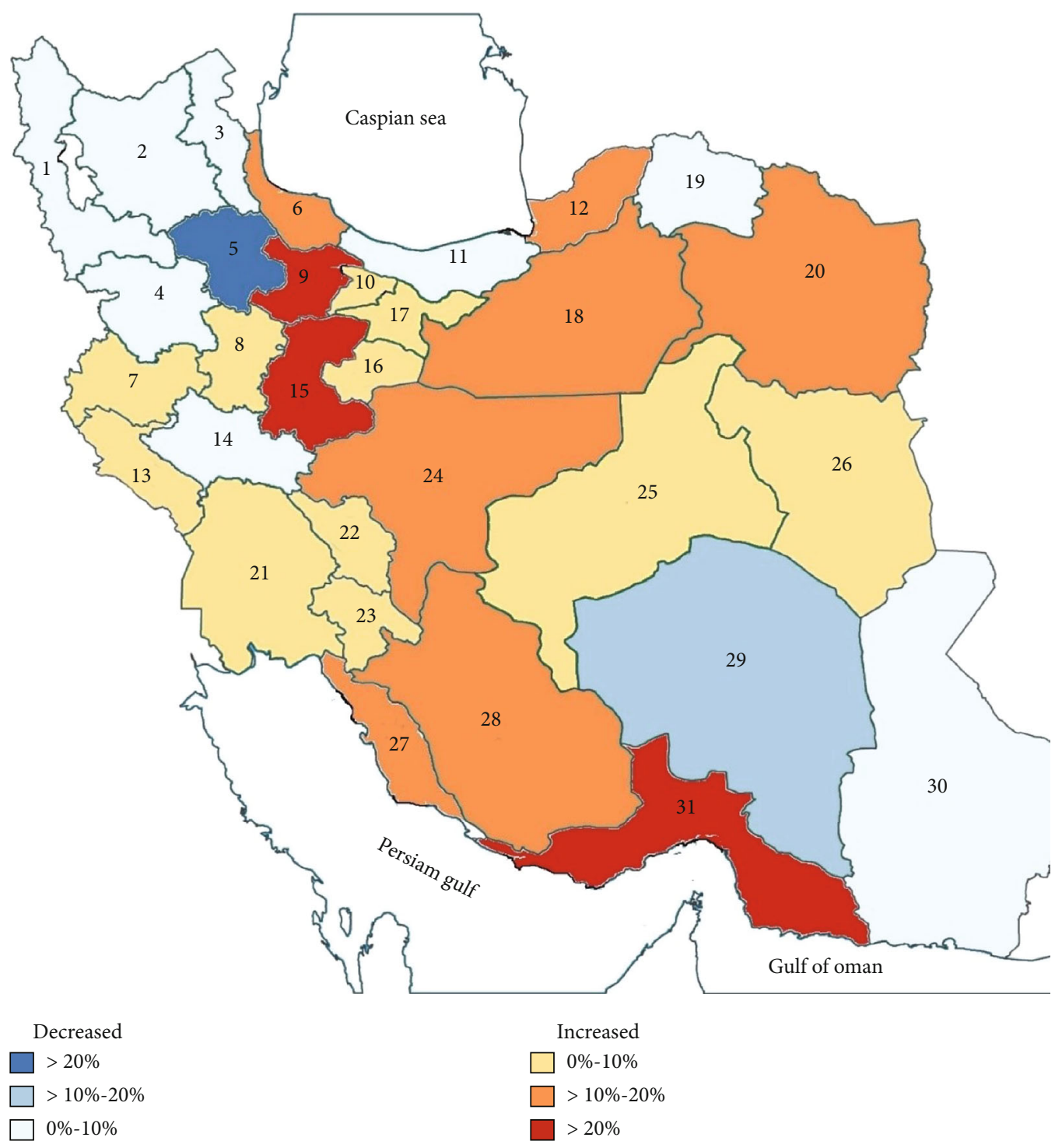

Figure 1: Relative changes in cancer age-standardized rate for both sexes from 2000 to 2016 in provinces of Iran. 1 indicates West Azarbayjan, 2 : East Azarbayjan, 3: Ardabil, 4: Kurdistan, 5: Zanjan, 6: Gilan, 7: Kermanshah, 8: Hamadan, 9: Qazvin, 10: Alborz, 11: Mazandaran, 12: Golestan, 13: Ilam, 14: Lorestan, 15: Markazi, 16: Qom, 17: Tehran, 18: Semnan, 19: North Khorasan, 20: Khorasan Razavi, 21: Khuzestan, 22: Chaharmahal and Bakhtiari, 23: Kohkiluye and Bouyerahmad, 24:Isfahan, 25: Yazd, 26: South Khorasan, 27: Boushehr, 28: Fars, 29: Kerman, 30: Sistan and Balouchestan, and 31: Hormozgan.

cases in Iran, as a high-middle SDI country [20], was similar to high-middle and high-SDI countries (Figure 3).

The provinces were ranked by their age-standardized rate (ASR) for each cancer group. This ranking enables comparing the rank of each province in the ASR of a specified cancer group with the rank of the province in the ASR of all cancers combined to find discrepancies (Figure 4). The comparison suggests that the distribution of some cancer groups does not follow the general distribution of cancer in Iran. For example, the fourth province in prostate cancer ASR and the second province in testicular cancer ASR was Zanjan which is the thirtieth province in cancer ASR. This indicates a surprisingly high incidence of prostate and testicular cancer in Zanjan. Sim- ilar examples are the high ASR of stomach cancer in Kohkiluyeh and Bouyerahmad, Mazandaran, Hamadan, and Ardabil; the high ASR of esophageal cancer in North, Razavi, and South Khorasan, Golestan, and Kurdistan; the high ASR of "Lip, oral cavity, and pharynx" cancer in Bushehr, and so forth.

3.2. The Pattern of Cancer Incidence by the Age Group. For childhood cancers (age $<14$ years), the three most common cancers excluding "other neoplasms" were lymphoid, hematopoietic, and related tissue; brain and nervous system; and liver cancers. In young- and middle-aged adults (age 15-54 years), the cancer groups that had the highest incidence were breast, colorectal, and brain and nervous system cancers. For 


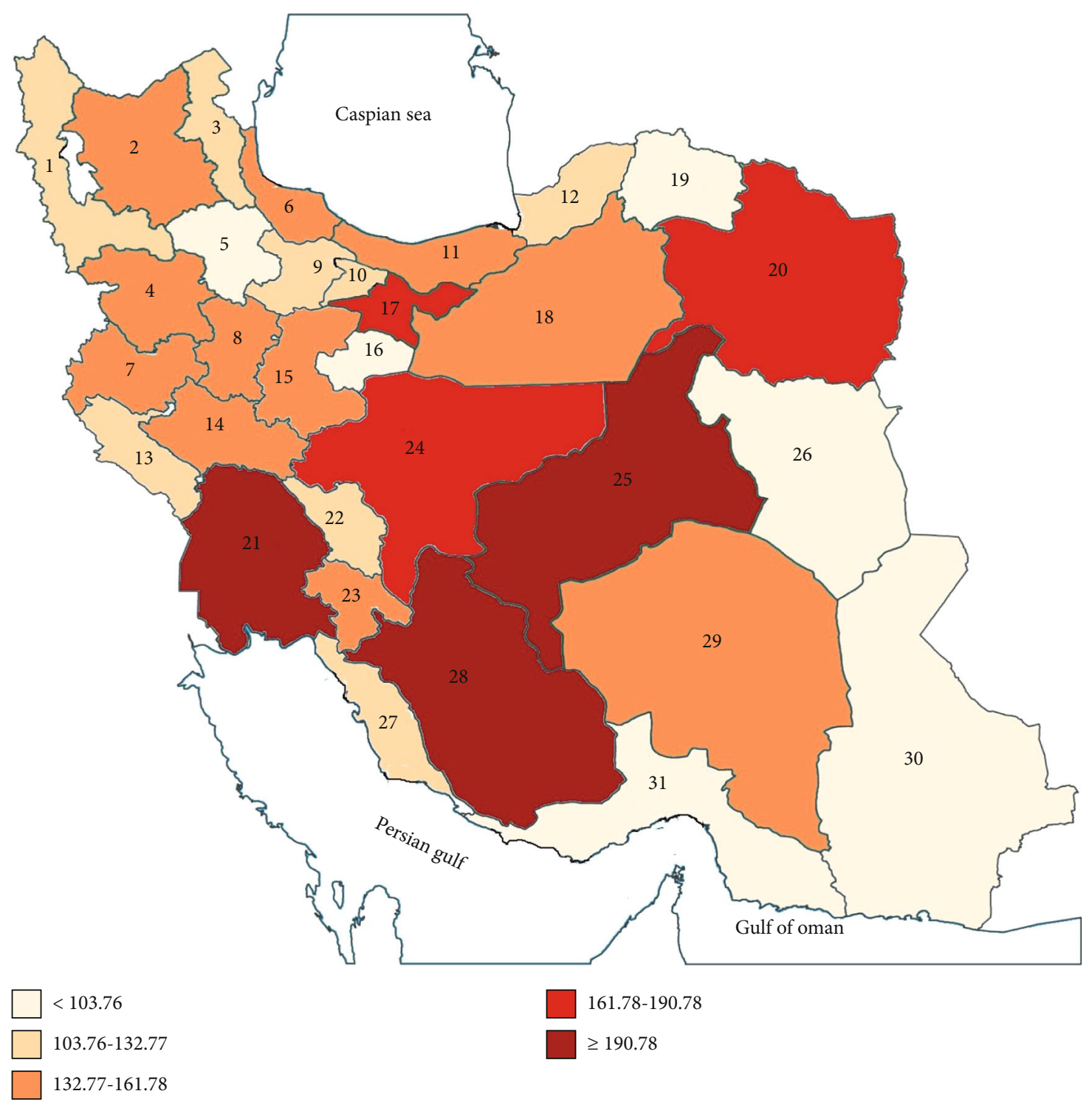

FIGURE 2: Cancer age-standardized rate (ASR) for both sexes in provinces of Iran in 2016. 1 indicates West Azarbayjan, 2: East Azarbayjan, 3: Ardabil, 4: Kurdistan, 5: Zanjan, 6: Gilan, 7: Kermanshah, 8: Hamadan, 9: Qazvin, 10: Alborz, 11: Mazandaran, 12: Golestan, 13: Ilam, 14: Lorestan, 15: Markazi, 16: Qom, 17: Tehran, 18: Semnan, 19: North Khorasan, 20: Khorasan Razavi, 21: Khuzestan, 22: Chaharmahal and Bakhtiari, 23: Kohkiluye and Bouyerahmad, 24:Isfahan, 25: Yazd, 26: South Khorasan, 27: Boushehr, 28: Fars, 29: Kerman, 30: Sistan and Balouchestan, and 31: Hormozgan.

the population older than 54 years, skin, breast, and colorectal cancers were the cancer groups with the highest number of incident cases (Figure 5).

3.3. The Cancers with the Highest Incidence in Iran in 2000 and 2016. The cancer groups with the highest crude rate in 2000 were skin, stomach, and breast cancer, whereas the cancer groups with the highest crude rate in 2016 were breast, skin, and colorectal cancer (Figure 6). Pancreatic, brain, and nervous system and liver cancers were the cancer groups that their ASR increased more than two times from 2000 to 2016. On the other side, esophageal; gallbladder and biliary tract; skin; lip, oral cavity, and pharynx; cervical; bladder; and stomach cancers were the cancer groups that their ASR decreased from 2000 to 2016 (Figure 6).

\section{Discussion}

We found that cancer incidence, crude rate, and agestandardized rate (ASR) increased in Iran from 2000 to 2016 with vast heterogeneity by cancer types, provinces, and sexes. Cancer incidence increased by $97 \%$ from 2000 to 2016 (Tables 1 and 2). Such an increase could be possibly due to the growth and aging of the population, improvements in the data recording system, and the increase in the prevalence of cancer risk factors [21-24]. Comparing percentage increases in incidence, crude rate, and ASR from 


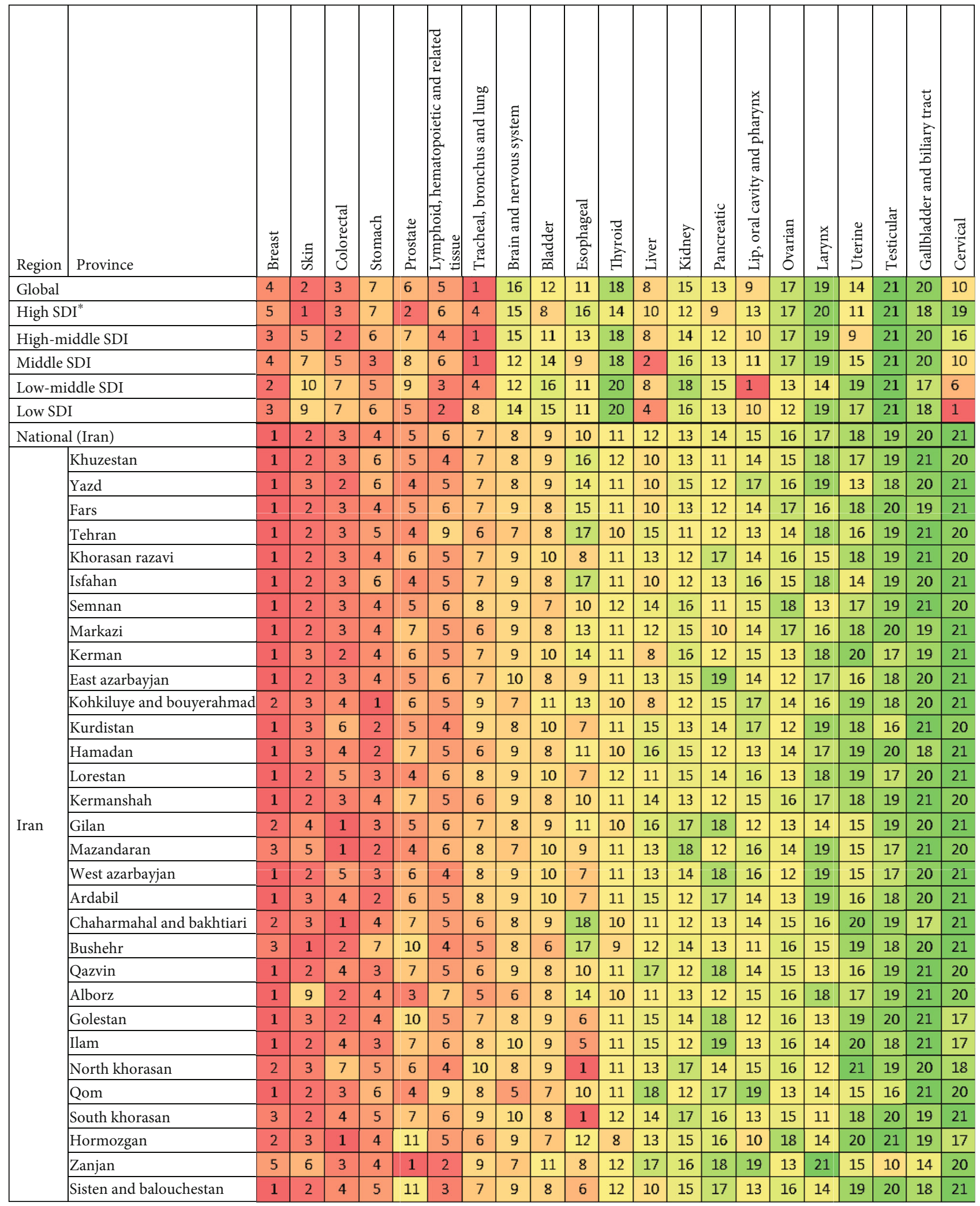

FIGURE 3: Cancers ranked by number of incident cases in both sexes, globally, by development status, nationally, and provincially in 2016. Colors correspond to the ranking (dark red is the cancer group with the most incidence, and dark green is the cancer group with the least incidence for the location indicated). The numbers inside each box indicate the ranking. The cancers were sorted by their ranking in national incidence. "Other cancers" group is not included in the ranking.*SDI: social development index. 


\begin{tabular}{|c|c|c|c|c|c|c|c|c|c|c|c|c|c|c|c|c|c|c|c|c|c|c|c|c|c|c|c|c|c|}
\hline Cancer group & 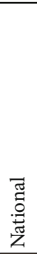 & 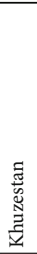 & 芯 & 莹 & 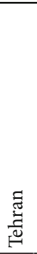 & 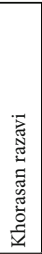 & 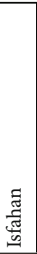 & $\begin{array}{l}\text { जี } \\
\text { हี } \\
\text { ڤ్ }\end{array}$ & $\begin{array}{l}\text { 䔍 } \\
\text { 莺 }\end{array}$ & 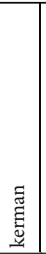 & 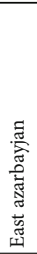 & 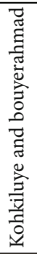 & 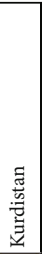 & 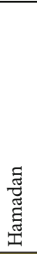 & 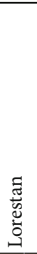 & 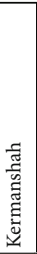 & $\frac{\sqrt{\pi}}{\overrightarrow{0}}$ & 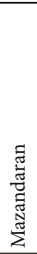 & 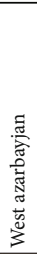 & 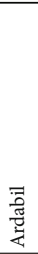 & 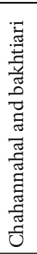 & $\begin{array}{l}\vec{z} \\
\tilde{\Xi} \\
\underline{n} \\
m\end{array}$ & $\begin{array}{l}\text { 芯 } \\
\text { वี }\end{array}$ & $\begin{array}{l}\text { No } \\
\stackrel{0}{Z}\end{array}$ & 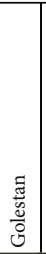 & $\underset{\exists}{\text { 寻 }}$ & 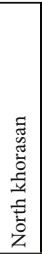 & घ̊ & 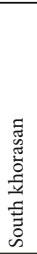 \\
\hline All cancers & 8 & 1 & 2 & 3 & 4 & 5 & 6 & 7 & 9 & 10 & 11 & 12 & 13 & 14 & 15 & 16 & 17 & 18 & 19 & 20 & 21 & 22 & 23 & 24 & 25 & 26 & 27 & 28 & 29 \\
\hline Breast & 8 & 2 & 3 & 5 & 1 & 6 & 4 & 7 & 9 & 18 & 11 & 20 & 13 & 14 & 15 & 10 & 16 & 25 & 17 & 22 & 24 & 21 & 19 & 12 & 27 & 26 & 28 & 23 & 30 \\
\hline Skin & 8 & 1 & 4 & 3 & 2 & 6 & 5 & 7 & 9 & 13 & 11 & 16 & 17 & 12 & 14 & 10 & 19 & 25 & 21 & 23 & 20 & 15 & 22 & 18 & 24 & 26 & 29 & 27 & 30 \\
\hline Colorectal & 8 & 2 & 1 & 3 & 4 & 13 & 6 & 9 & 15 & 12 & 11 & 18 & 21 & 16 & 22 & 17 & 5 & 7 & 23 & 24 & 14 & 10 & 20 & 26 & 19 & 27 & 31 & 29 & 28 \\
\hline Stomach & 15 & 8 & 20 & 3 & 16 & 7 & 22 & 14 & 19 & 11 & 9 & 1 & 6 & 4 & 13 & 23 & 10 & 2 & 12 & 5 & 17 & 30 & 18 & 27 & 21 & 24 & 25 & 31 & 29 \\
\hline Prostate & 11 & 3 & 2 & 8 & 1 & 9 & 5 & 13 & 22 & 14 & 12 & 17 & 6 & 21 & 10 & 24 & 20 & 7 & 15 & 19 & 26 & 30 & 25 & 16 & 28 & 27 & 23 & 18 & 29 \\
\hline Lymphoid, hematopoietic and related tissue & 14 & 1 & 3 & 4 & 18 & 6 & 10 & 17 & 13 & 7 & 11 & 5 & 2 & 15 & 8 & 19 & 20 & 12 & 9 & 16 & 22 & 27 & 21 & 30 & 25 & 26 & 24 & 28 & 29 \\
\hline Tracheal, bronchus and lung & 9 & 1 & 3 & 2 & 4 & 5 & 6 & 15 & 8 & 13 & 7 & 25 & 12 & 10 & 17 & 11 & 16 & 21 & 14 & 20 & 22 & 18 & 23 & 19 & 24 & 26 & 29 & 27 & 31 \\
\hline Brain and nervous system & 9 & 1 & 2 & 4 & 3 & 6 & 5 & 7 & 11 & 13 & 10 & 15 & 8 & 19 & 14 & 20 & 16 & 12 & 18 & 17 & 24 & 27 & 22 & 21 & 28 & 29 & 25 & 23 & 30 \\
\hline Esophageal & 15 & 21 & 22 & 23 & 26 & 2 & 29 & 13 & 24 & 27 & 10 & 20 & 3 & 18 & 8 & 14 & 17 & 11 & 6 & 9 & 31 & 32 & 19 & 28 & 4 & 7 & 1 & 25 & 5 \\
\hline Thyroid & 12 & 3 & 2 & 1 & 4 & 6 & 14 & 9 & 10 & 11 & 15 & 7 & 22 & 13 & 19 & 21 & 5 & 8 & 23 & 20 & 16 & 17 & 24 & 25 & 18 & 28 & 29 & 30 & 26 \\
\hline Liver & 10 & 1 & 3 & 5 & 14 & 9 & 6 & 12 & 8 & 2 & 20 & 4 & 22 & 19 & 7 & 16 & 24 & 21 & 17 & 25 & 13 & 15 & 31 & 11 & 29 & 28 & 23 & 30 & 27 \\
\hline Kidney & 8 & 1 & 9 & 5 & 2 & 3 & 4 & 22 & 10 & 25 & 23 & 6 & 13 & 16 & 14 & 7 & 27 & 28 & 18 & 17 & 11 & 20 & 15 & 19 & 24 & 12 & 30 & 21 & 31 \\
\hline Pancreatic & 11 & 1 & 4 & 2 & 10 & 16 & 6 & 3 & 5 & 8 & 31 & 13 & 18 & 7 & 15 & 9 & 23 & 19 & 26 & 22 & 17 & 12 & 25 & 14 & 27 & 28 & 20 & 24 & 29 \\
\hline Lip, oral cavity and pharynx & 11 & 2 & 4 & 1 & 3 & 6 & 12 & 7 & 8 & 17 & 19 & 15 & 27 & 10 & 25 & 13 & 9 & 24 & 28 & 23 & 16 & 5 & 21 & 22 & 14 & 26 & 29 & 31 & 20 \\
\hline Ovarian & 14 & 3 & 4 & 8 & 5 & 10 & 9 & 17 & 13 & 12 & 15 & 1 & 2 & 11 & 7 & 16 & 20 & 21 & 6 & 19 & 18 & 24 & 23 & 26 & 29 & 27 & 28 & 25 & 30 \\
\hline Larynx & 14 & 5 & 12 & 3 & 10 & 4 & 13 & 1 & 7 & 18 & 27 & 9 & 30 & 19 & 26 & 17 & 15 & 28 & 31 & 29 & 22 & 16 & 11 & 25 & 8 & 20 & 6 & 23 & 2 \\
\hline Uterine & 8 & 3 & 1 & 6 & 4 & 14 & 2 & 5 & 21 & 20 & 11 & 24 & 13 & 18 & 19 & 22 & 9 & 7 & 10 & 12 & 29 & 25 & 17 & 15 & 26 & 27 & 31 & 16 & 28 \\
\hline Testicular & 13 & 3 & 1 & 18 & 8 & 11 & 9 & 4 & 22 & 7 & 14 & 17 & 6 & 21 & 12 & 23 & 19 & 5 & 10 & 15 & 24 & 29 & 26 & 20 & 28 & 25 & 27 & 16 & 30 \\
\hline Gallbladder and biliary tract & 11 & 1 & 5 & 3 & 12 & 17 & 6 & 19 & 8 & 4 & 23 & 10 & 14 & 2 & 15 & 13 & 21 & 26 & 18 & 20 & 9 & 22 & 27 & 32 & 30 & 24 & 16 & 29 & 28 \\
\hline Cervical & 12 & 1 & 3 & 4 & 6 & 2 & 11 & 10 & 16 & 17 & 26 & 23 & 13 & 18 & 19 & 14 & 28 & 22 & 25 & 24 & 27 & 29 & 20 & 15 & 7 & 5 & 9 & 21 & 30 \\
\hline
\end{tabular}

FIGURE 4: Iran's provinces ranked by age-standardized rate (ASR) in both sexes for each cancer group ${ }^{1}, 2016 .{ }^{1}$ National ASR is also included in the first column for better comparison. Colors correspond to the ranking (dark red is the cancer group with the most incidence, and dark green is the cancer group with the least incidence for cancer indicated). The numbers inside each box indicate the ranking. The provinces were sorted based on their 2016 ASR in Iran. "Other cancers" group is not included in the ranking.

2000 and 2016 reveals that population growth accounts for $31 \%$, and population aging accounts for $55 \%$ out of $97 \%$ increase in cancer incidence. Therefore, the remaining $11 \%$ could be attributed to other causes such as improvements in the data recording system and the increase in the prevalence of cancer risk factors. Notably, this attribution was calculated according to all cancer types' incidence and could not be generalized to each defined cancer group. Considering the projected aging of Iran's population in the following years, a further increase in cancer incidence is expected, which would be a significant health concern $[25,26]$.

The study estimates that the cancer ASR using the world standard population was 147.9 for women and 159.5 per 100,000 people for men in Iran in 2016. This ASR is lesser than the global cancer ASR estimated by the GBD study in 2016 (213.9 per 100,000 people for women and 306.8 per 100,000 people for men) [2] and the GLOBOCAN study in 2018 (182.6 per 100,000 people for women and 218.6 for men) [27]. The difference in ASR estimates could be due to the incompleteness of the cancer registry program in Iran $[9,11]$. Upcoming sections will discuss study results for some cancer groups.

4.1. Breast Cancer. It was shown that breast cancer had the highest incidence among cancer groups in Iran and most of its provinces, which was in accordance with some previous studies [28]. However, breast cancer is not the most common cancer in the world or any SDI quintiles $[2,27]$. This differ- ence indicates a disproportionate high incidence of breast cancer in Iran.

4.2. Skin Cancer. Skin cancer (including melanoma and non-melanoma skin cancers) ranked second by incidence in Iran. Besides, this cancer was the most common cancer type in the older adults and elderly (age $>54$ years) (Figure 5). Although a previous study introduced Qom as a hot spot for skin cancer in 2009, our results showed a low level of skin cancer ASR in this province [29]. Moreover, it was shown that the ranking of provinces by skin cancer ASR was similar to the pattern of cancer ASR in Iran (Figure 4).

4.3. Colorectal Cancer. Colorectal cancer had the third highest ASR in 2016, which shows a $41 \%$ increase since 2000 . Such an increase in colorectal cancer incidence could be partly due to changes in the dietary habits of the Iranian population, lower consumption of vegetables and fruits and higher intake of red meat, and the community transition to a sedentary lifestyle $[24,30]$. Furthermore, it was surprisingly shown that colorectal cancer incidence was higher in women than men in 2016. This higher incidence shows a sex transition in the incidence pattern of this cancer in Iran [24, 31]. We found that Mazandaran, Gilan, and Boushehr had high ASR in colorectal cancer in proportion to their all cancer types' ASR (Figures 3 and 4). The unexpected high ASR of colorectal cancer in Gilan and Mazandaran ws known before [30]; however, most of the previous studies did not mention 


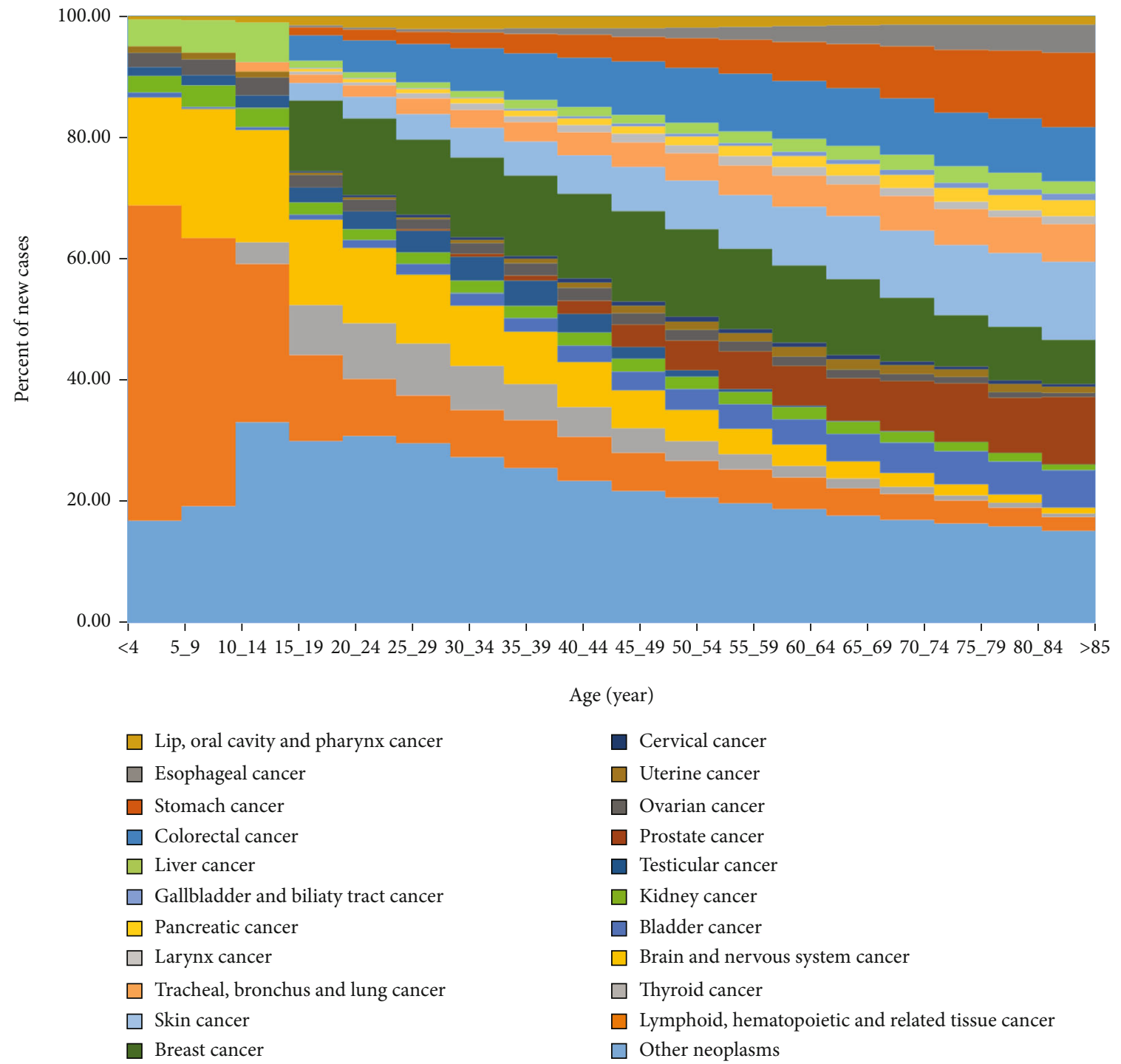

FIgURe 5: Age-specific contributions of cancer types to total cancer incidence for both sexes, 2016. For International Classification of Disease codes pertaining to each neoplasm group, see eTbale 1 in the supplement.

Boushehr as a province with unexpected high colorectal cancer ASR. High prevalence of obesity and inflammatory bowel disease (IBD) in Mazandaran and Gilan and low physical activity in Boushehr might be the risk factors for the mentioned unexpected high ASR of colorectal cancer in these provinces [32-35].

4.4. Stomach Cancer. Stomach cancer was the fourth cancer by incidence in 2016. It was shown that there is a disproportionate high ASR of this cancer in the western and northern provinces of Iran (Kohkiluye and Bouyerahmad, Kurdistan, and Hamadan, Gilan, Mazandaran, and Ardabil) (Figure 4). Previous studies also determined a similar epidemiologic distribution [36, 37]; however, they did not recognize Kohkiluye and Bouyerahamd as the province with the highest ASR for stomach cancer. A combination of $\mathrm{H}$. pylori infection, gastroesophageal reflux disease, smoking, and heavy metals was reported as risk factors of gastric cancer in these provinces [38-40].
4.5. Prostate Cancer. Prostate cancer was the tenth and fifth cancer by incidence in 2000 and 2016, respectively. The cancer ASR increased by $191 \%$ during this period (Figure 6). Such a sharp increase could be due to the urbanization of the country, which is accompanied with increasing high-fat diet consumption, decreasing energy expenditure and physical activity, higher levels of pollution, more contact with carcinogens, and higher cancer detection rates [41, 42]. It was shown that Tehran, Yazd, and Khuzestan were the provinces with the highest prostate cancer ASR. Besides, Zanjan, Kurdistan, and Mazandaran showed high ASR for prostate cancer in comparison with their all cancer types' ASR (Figure 4). Accordingly, a previous study demonstrated that the relative risk for prostate cancer after adjusting its risk factors was much higher in Zanjan, Kurdistan, and Mazandaran provinces than the average national level [42].

4.6. Esophageal Cancer. This study, following some other studies, showed that esophageal cancer incidence is 


\begin{tabular}{|c|c|c|c|}
\hline \multicolumn{2}{|r|}{2000} & \multicolumn{2}{|l|}{2016} \\
\hline Rank & Cancer & Cancer & Rank \\
\hline 1 & Skin & Breast & 1 \\
\hline 2 & Stomach & Skin & 2 \\
\hline 3 & Breast & Colorectal & 3 \\
\hline 4 & Bladder & Stomach & 4 \\
\hline 5 & Colorectal & Prostate & 5 \\
\hline 6 & Esophageal & $\begin{array}{l}\text { Lymphoid, hematopoietic } \\
\text { and related tissue }\end{array}$ & 6 \\
\hline 7 & $\begin{array}{l}\text { Lymphoid, hematopoietic } \\
\text { and related tissue }\end{array}$ & Tracheal, bronchus and lung & 7 \\
\hline 8 & Lip, oral cavity and pharynx & $\begin{array}{l}\text { Brain and nervous system } \\
\text { cancer }\end{array}$ & 8 \\
\hline 9 & Tracheal, bronchus and lung & Bladder & 9 \\
\hline 10 & Prostate & Esophageal & 10 \\
\hline 11 & $\begin{array}{l}\text { Brain and nervous system } \\
\text { cancer }\end{array}$ & Thyroid & 11 \\
\hline 12 & Larynx & Liver & 12 \\
\hline 13 & Kidney & Kidney & 13 \\
\hline 14 & Thyroid & Pancreatic & 14 \\
\hline 15 & Gallbladder and biliary tract & Lip, oral cavity and pharynx & 15 \\
\hline 16 & Ovarian & Ovarian & 16 \\
\hline 17 & Cervical & Larynx & 17 \\
\hline 18 & Liver & Uterine & 18 \\
\hline 19 & Uterine & Testicular & 19 \\
\hline 20 & Testicular & Gallbladder and biliary tract & 20 \\
\hline 21 & Pancreatic & Cervical & 21 \\
\hline
\end{tabular}

\begin{tabular}{|c|c|}
\hline $\begin{array}{c}\text { Change in } \\
\mathrm{CR}^{\mathrm{a}}, \%\end{array}$ & $\begin{array}{c}\text { Change in } \\
\text { ASR }^{\mathrm{b}}, \%\end{array}$ \\
\hline 117 & 42 \\
\hline-16 & -44 \\
\hline 108 & 41 \\
\hline-7 & -37 \\
\hline 311 & 191 \\
\hline 81 & 48 \\
\hline 214 & 104 \\
\hline 334 & 270 \\
\hline-11 & -39 \\
\hline-36 & -58 \\
\hline 173 & 106 \\
\hline 283 & 236 \\
\hline 93 & 45 \\
\hline 753 & 467 \\
\hline-17 & -42 \\
\hline 97 & 40 \\
\hline 24 & -14 \\
\hline 175 & 76 \\
\hline 129 & 70 \\
\hline-18 & -47 \\
\hline-5 & -40 \\
\hline
\end{tabular}

FIGURE 6: Cancers ranked for both sexes by national crude rate, including the percentage change in crude rate and the percentage change in the age-standardized rate between 2000 and 2016. The "other cancer" group is not included here because it contains multiple different types of cancers. Solid lines connecting the 2000 and 2016 charts indicate increased or unchanged rank for the connected cancers; dotted lines indicated decreased rank. ${ }^{\mathrm{a}} \mathrm{CR}$ : crude rate; ${ }^{\mathrm{b}} \mathrm{ASR}$, age-standardized rate.

decreasing in Iran $[41,43]$. This cancer was the tenth cancer by incidence in 2016 and was the first cancer by incidence in South and North Khorasan (Figure 3). North, Razavi, and South Khorasan, Golestan, and Kurdistan were the provinces with the highest ASR for this cancer (Figure 4). These results approved previous reports, indicating that these provinces have one of the highest reported rates of esophageal squamous cell carcinoma worldwide. Most of the aforementioned provinces have common borders with Turkmenistan and Afghanistan countries that have high ASR for esophageal cancer due to their susceptible genetic/ethnic background [44-46]. Besides genetic susceptibility, exposure to polycyclic aromatic hydrocarbons (from opium smoking and indoor air pollution), drinking hot tea, low intake of fruits and vegetables, and drinking unpiped water were determined as risk factors for esophageal cancer in these areas $[47,48]$.

4.7. Tracheal, Bronchus, and Lung Cancer. The tracheal, bronchus, and lung cancer group had the highest cancer incidence worldwide in 2016; however, it was the seventh cancer by incidence in Iran. Therefore, this cancer showed one of the most prominent differences in ranking of cancer types by incidence between Iran and other countries of the world (Figure 3). It was shown that the ranking of provinces by this cancer ASR was majorly similar to the pattern of cancer ASR in Iran, having the highest ASRs in Khuzestan, Yazd, and Fars (Figure 4). Surprisingly, these provinces were not the highest in cigarette and water-pipe smoking prevalence among provinces of Iran [49]. However, these provinces, especially Khuzestan and Fars, along with other provinces with high ASRs like Tehran, Isfahan, and Khorasan Razavi, had the highest premature deaths attributable to ambient particulate matter 2.5 in 2016 [50]. Provinces like West and East Azarbayjan, Hamadan, and Boushehr had higher ranks in the tracheal, bronchus, and lung cancer ASR in comparison with their ranks in all cancers ASR, which could be attributed to the high prevalence of smoking and high concentration of air pollutions [50,51] (Figure 4).

4.8. Limitations. We faced some limitations in defining cancer groups due to the lack of data for some subgroups. For example, Hodgkin lymphoma, non-Hodgkin lymphoma, leukemia, and multiple myeloma had not been specified in the data. Therefore, the "hematopoietic, lymphoid, and 
related tissue" group was defined, which includes all the four mentioned cancer subgroups. Another example was melanoma and nonmelanoma skin cancers, and the "skin cancer" group was defined to cover both subgroups.

\section{Conclusion}

We showed that cancer incidence, crude rate, and agestandardized rate (ASR) in Iran increased from 2000 to 2016 with vast heterogeneity by cancer type, province, and sex. It was also shown that the crude rate of cancer in Iran was 155 per 100,000 people for both sexes combined in 2016, much less than the global cancer crude rate estimated by the GBD study in 2016 and the GLOBOCAN study in 2018. This study represents quantitative data on different cancer types' incidence in Iran's provinces, which would help the appropriate allocation of resources to each part of the health care system and the development of effective national cancer control plans $[4,13]$.

\section{Data Availability}

The datasets used and/or analyzed during the current study are available from the corresponding author on reasonable request. Besides, data are available from https://vizit.report/ en/dashboard.html

\section{Conflicts of Interest}

The authors declare that they have no competing interests.

\section{Authors' Contributions}

JK extracted, analyzed, and visualized data and wrote the manuscript. A-AK supervised the study and revised the manuscript. All authors read and approved the final manuscript.

\section{Acknowledgments}

This study was supported by the Social Determinants of Health Research Center, Shahid Beheshti University of Medical Sciences.

\section{Supplementary Materials}

eTable 1: list of International Classification of Disease (ICD) codes mapped to the article cause list for cancer. (Supplementary Materials)

\section{References}

[1] M. Quaresma, M. P. Coleman, and B. Rachet, "40-year trends in an index of survival for all cancers combined and survival adjusted for age and sex for each cancer in England and Wales, 1971-2011: A population-based study," Lancet, vol. 385, no. 9974, pp. 1206-1218, 2015.

[2] C. Fitzmaurice, T. F. Akinyemiju, F. H. Al Lami et al., "Global, regional, and national cancer incidence, mortality, years of life lost, years lived with disability, and disabilityadjusted life-years for 29 cancer groups, 1990 to 2016 a systematic analysis for the global burden of disease study global burden o," JAMA Oncology, vol. 4, no. 11, pp. 1553-1568, 2018.

[3] C. Allemani, T. Matsuda, V. Di Carlo et al., "Global surveillance of trends in cancer survival 2000-14 (CONCORD-3): analysis of individual records for 37513025 patients diagnosed with one of 18 cancers from 322 population-based registries in 71 countries," Lancet, vol. 391, no. 10125, pp. 10231075, 2018.

[4] C. Fitzmaurice, D. Abate, N. Abbasi et al., "Global, regional, and national cancer incidence, mortality, years of life lost, years lived with disability, and disability-adjusted life-years for 29 cancer groups, 1990 to 2017: a systematic analysis for the global burden of disease study," JAMA Oncology, vol. 5, no. 12, pp. 1749-1768, 2019.

[5] W. Wei, H. Zeng, R. Zheng et al., "Cancer registration in China and its role in cancer prevention and control," The Lancet Oncology, vol. 21, p. e342, 2020.

[6] E. Mohebbi, A. Nahvijou, M. Hadji et al., "Iran cancer statistics in 2012 and projection of cancer incidence by 2035," Basic \& Clinical Cancer Research, vol. 9, no. 3, pp. 3-22, 2017.

[7] G. Roshandel, A. Ghanbari-Motlagh, E. Partovipour et al., "Cancer incidence in Iran in 2014: results of the Iranian national population-based cancerr," Cancer Epidemiology, vol. 61, pp. 50-58, 2019.

[8] A. Sheikhtaheri, A. Nahvijou, Z. Sedighi et al., "Development of a tool for comprehensive evaluation of population-based cancer registries," International Journal of Medical Informatics, vol. 117, pp. 26-32, 2018.

[9] G. Mohammadi, M. E. Akbari, Y. Mehrabi, and A. G. Motlagh, "Quality assessment of the national cancer registry in iran: completeness and validity," Iranian Journal of Cancer Prevention, vol. 9, no. 6, 2016.

[10] R. Amirkhah, H. Naderi-Meshkin, M. Mirahmadi, A. Allahyari, and H. R. Sharifi, "Cancer statistics in Iran: towards finding priority for prevention and treatment," Cancer Press, vol. 3, no. 2, p. 27, 2017.

[11] G. Mohammadi, M. E. Akbari, Y. Mehrabi et al., "Estimating completeness of cancer registration in Iran with capturerecapture methods," Asian Pacific Journal of Cancer Prevention, vol. 17, no. S3, pp. 93-99, 2016.

[12] A. Etemadi, A. Sadjadi, S. Semnani, S. M. Nouraie, H. Khademi, and M. Bahadori, "Cancer registry in Iran: a brief overview," Archives of Iranian Medicine, vol. 11, no. 5, pp. 577-580, 2008.

[13] C. Fitzmaurice, D. Dicker, A. Pain et al., "The global burden of cancer 2013," JAMA Oncology, vol. 1, no. 4, pp. 505-527, 2015.

[14] Cancer, Tehran: NCDRC, Tehran University of Medical Sciences, 2016, https://vizit.report/en/dashboard.html.

[15] Cancer Office, The Ministry of Health and Medical Education, The Report of The National Cancer Registry Program, 2015, https://sghc.iums.ac.ir/files/sghc/files/pdf/Gozaresh_ Keshvari_Sabte_Saratan_1394_-_Sent_(1).pdf.

[16] M. Modirian, S. Rahimzadeh, Z. Cheraghi et al., "Quality evaluation of national cancer registry system in Iran: study protocol," Archives of Iranian Medicine, vol. 17, no. 3, pp. 193-197, 2014.

[17] University of Washington, Socio-demographic Index (SDI) | Institute for Health Metrics and Evaluation, Institute for health Metrics and Evaluation, 2019.

[18] N. Rajai, A. Ghanbari, M. Yoosefi et al., "National and subnational trends in incidence and mortality of lung cancer in Iran 
from 1990 to 2016," Asia-Pacific Journal of Clinical Oncology, vol. 16, no. 3, pp. 129-136, 2020.

[19] M. Shabani, S. Saeedi Moghaddam, B. Ataeinia et al., "Trends of national and subnational incidence of childhood cancer groups in Iran: 1990-2016," Frontiers in Oncology, vol. 9, 2020.

[20] Global Burden of Disease Collaborative Network, Global Burden of Disease Study 2016 (GBD 2016) Socio-Demographic Index (SDI) 1970-2016, Institute for Health Metrics and Evaluation (IHME), 2016.

[21] F. Mansour-Ghanaei, G. Varshi, F. Joukar et al., "Prevalence of pre-cancerous colon lesions in referred patients under patronage of a local relief foundation in Guilan province," Journal of Medicine and Life, vol. 12, no. 2, pp. 133-139, 2019.

[22] L. A. Torre, F. Bray, R. L. Siegel, J. Ferlay, J. Lortet-Tieulent, and A. Jemal, "Global cancer statistics, 2012," CA: a Cancer Journal for Clinicians, vol. 65, no. 2, pp. 87-108, 2015.

[23] G. Roshandel, S. Semnani, A. Fazel et al., "Building cancer registries in a lower resource setting: the 10-year experience of Golestan, Northern Iran," Cancer epidemiology, vol. 52, pp. 128-133, 2018.

[24] E. Abdifard, S. Ghaderi, S. Hosseini, and M. Heidari, "Incidence trends of colorectal cancer in the west of Iran during 2000-2005," Asian Pacific Journal of Cancer Prevention, vol. 14, no. 3, pp. 1807-1811, 2013.

[25] M. Basakha, K. Yavari, H. Sadeghi, and A. Naseri, "Health care cost disease as a threat to iranian aging society," Journal of research in health sciences, vol. 14, no. 2, pp. 152-156, 2014.

[26] P. Azadi and M. Mesgaran, Farzaneh Roudi Former Director of MENA Program at the Population Reference Bureau Iran's Population Dynamics and Demographic Window of Opportunity, 2017.

[27] F. Bray, J. Ferlay, I. Soerjomataram, R. L. Siegel, L. A. Torre, and A. Jemal, "Global cancer statistics 2018: GLOBOCAN estimates of incidence and mortality worldwide for 36 cancers in 185 countries," CA: a Cancer Journal for Clinicians, vol. 68, no. 6, pp. 394-424, 2018.

[28] Z. Almasi, A. Mohammadian-Hafshejani, and H. Salehiniya, "Incidence, mortality, and epidemiological aspects of cancers in Iran; differences with the world data," Journal of BUON, vol. 21, no. 4, pp. 994-1004, 2016.

[29] R. Pakzad, M. Ghoncheh, Z. Pournamdar et al., "Spatial analysis of skin cancer incidence in Iran," Asian Pacific Journal of Cancer Prevention, vol. 17, no. S3, pp. 33-37, 2020.

[30] R. Pakzad, A. Moudi, Z. Pournamdar et al., "Spatial analysis of colorectal cancer in Iran," Asian Pacific Journal of Cancer Prevention, vol. 17, no. S3, pp. 53-57, 2016.

[31] S. Hasanpour-Heidari, A. Fazel, S. Semnani et al., "Temporal and geographical variations in colorectal cancer incidence in Northern Iran 2004-2013," Cancer Epidemiology, vol. 59, pp. 143-147, 2019.

[32] M. Sahebkar, H. H. Miri, P. Noormohammadpour et al., "Geographical patterning of physical activity prevalence in Iran: Spatial analysis of 4 pooled national health surveys among 119,560 adults," Journal of Physical Activity and Health, vol. 16, no. 12, pp. 1071-1077, 2019.

[33] M. M. Malekzadeh, H. Vahedi, K. Gohari et al., "Emerging epidemic of inflammatory bowel disease in a middle income country: a nation-wide study from Iran," Archives of Iranian Medicine, vol. 19, no. 1, 2016.

[34] B. Mahaki, Y. Mehrabi, A. Kavousi, and V. J. Schmid, "Joint spatio-temporal shared component model with an application in Iran Cancer Data," Asian Pacific journal of cancer prevention: APJCP, vol. 19, no. 6, pp. 1553-1560, 2018.

[35] M. Ahmadi, M. Moosazadeh, H. M.-E. Vardanjani, and A. Dehghan, "Prevalence of obesity and overweight and their related factors among the adults of Mazandaran Province, Iran, in 2010," Electronic Physician, vol. 6, no. 4, pp. 95595561, 2014.

[36] A. Zahedi, H. Rafiemanesh, M. Enayatrad, M. Ghoncheh, and H. Salehiniya, "Incidence, trends and epidemiology of cancers in North West of Iran," Asian Pacific journal of cancer prevention: APJCP, vol. 16, no. 16, pp. 7189-7193, 2015.

[37] R. Pakzad, Y. Khani, I. Pakzad et al., "Spatial analysis of stomach cancer incidence in Iran," Asian Pacific journal of cancer prevention: APJCP, vol. 17, no. S3, pp. 27-32, 2016.

[38] O. Eskandari, M. Ghias, A. Fatehizadeh, M. Zare, M. Amin, and A. Kazemi, "Geographical distribution of stomach cancer related to heavy metals in Kurdistan, Iran," International Journal of Environmental Health Engineering, vol. 4, no. 1, p. 12, 2015.

[39] Gastric cancer in Ardabil, Iran-a review and update on cancer registry data - PubMed, 2020, https://pubmed.ncbi.nlm.nih .gov/21039022/.

[40] B. D. Aguemon, M. J. Struelens, A. Massougbodji, and E. M. Ouendo, "Prevalence and risk-factors for Helicobacter pylori infection in urban and rural Beninese populations," Clinical Microbiology and Infection, vol. 11, no. 8, pp. 611-617, 2005.

[41] S. Momenyan, M. Sadeghifar, F. Sarvi et al., "Relationship between urbanization and cancer incidence in Iran using quantile regression," Asian Pacific Journal of Cancer Prevention, vol. 17, no. S3, pp. 113-117, 2016.

[42] A. Haddad-Khoshkar, T. Jafari-Koshki, and B. Mahaki, "Investigating the incidence of prostate cancer in Iran 2005-2008 using Bayesian spatial ecological regression models," Asian Pacific Journal of Cancer Prevention, vol. 16, no. 14, pp. 5917-5921, 2015.

[43] H. Mehdizadeh, G. Mahmoudi, D. Moslemi, A. Bijani, and M. A. Jahani, "A 25-year trend in gastrointestinal cancers in northern Iran (1991-2016)," Caspian Journal of Internal Medicine, vol. 10, pp. 396-401, 2019.

[44] S. J. Pournaghi, S. K. Hojjat, F. Barazandeh Noveyri et al., "Tobacco consumption, opium use, alcohol drinking and the risk of esophageal cancer in North Khorasan, Iran," Journal of Substance Use, vol. 24, no. 1, pp. 105-109, 2019.

[45] H. Salehiniya, S. Hassanipour, F. Mansour-Ghanaei et al., "The incidence of esophageal cancer in Iran: a systematic review and meta-analysis," Biomedical Research and Therapy, vol. 5, no. 7, pp. 2493-2503, 2018.

[46] R. Moradzadeh, P. Golmohammadi, B. Ghaitasi, H. Nadrian, and A. Najafi, "Incidence of esophageal cancer in Iran, a population-based study: 2001-2015," Journal of Gastrointestinal Cancer, vol. 50, no. 3, pp. 507-512, 2019.

[47] M. Gholipour, F. Islami, G. Roshandel et al., "Esophageal cancer in Golestan province, Iran: a review of genetic susceptibility and environmental risk factors," Middle East journal of digestive diseases, vol. 8, no. 4, pp. 249-266, 2016.

[48] M. Sheikh, H. Poustchi, A. Pourshams et al., "Individual and combined effects of environmental risk factors for esophageal cancer based on results from the Golestan cohort study," Gastroenterology, vol. 156, no. 5, pp. 1416-1427, 2019.

[49] S. Nemati, A. Rafei, N. D. Freedman, A. Fotouhi, F. Asgary, and K. Zendehdel, "Cigarette and water-pipe use in Iran: 
Geographical distribution and time trends among the adult population; a pooled analysis of national STEPS surveys, 2006-2009," Archives of Iranian Medicine, vol. 20, no. 5, pp. 295-301, 2017.

[50] M. Shamsipour, M. S. Hassanvand, K. Gohari et al., "National and sub-national exposure to ambient fine particulate matter (PM2.5) and its attributable burden of disease in Iran from 1990 to 2016," Environmental Pollution, vol. 255, Part 1, 2019.

[51] A. Meysamie, R. Ghaletaki, M. Haghazali et al., "Pattern of tobacco use among the iranian adult population: results of the national Survey of Risk Factors of Non-Communicable Diseases (SuRFNCD-2007)," Tobacco Control, vol. 19, no. 2, pp. 125-128, 2010. 